\title{
Dyke-Davidoff-Masson syndrome with crossed cerebellar atrophy
}

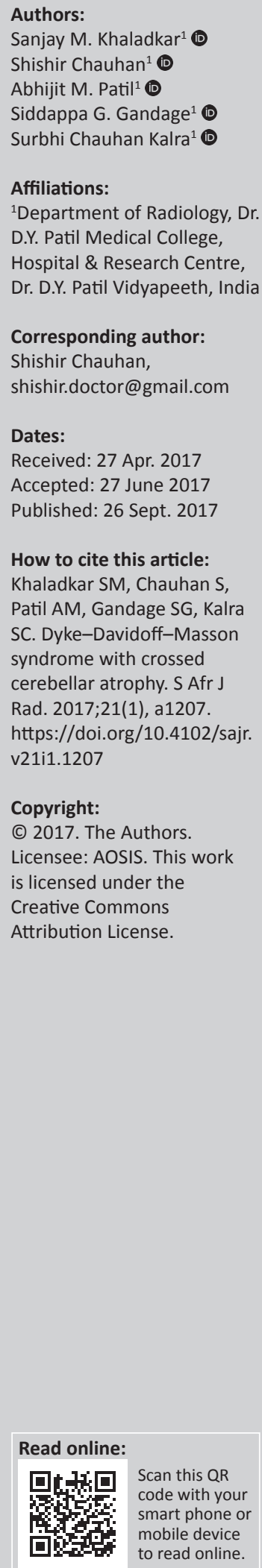

Dyke-Davidoff-Masson syndrome is a rare condition with classical, clinical and radiological changes - mental retardation, hemiparesis, facial asymmetry, seizures and cerebral hemiatrophy with calvarial changes. Contralateral cerebellar atrophy is rare and occurs if insult occurs after 1 month of age. We report a case of a 6-year-old female child presenting with right-sided hemiparesis, convulsions and left cerebral hemiatrophy with an old infarct in left middle cerebral artery (MCA) territory, ipsilateral calvarial thickening and right (crossed) cerebellar atrophy.

\section{Introduction}

Dyke-Davidoff-Masson syndrome (DDMS) is a rare disease characterised by cerebral atrophy in one cerebral hemisphere because of an insult in utero or in early childhood (acquired cases) characterised by hemiparesis, seizures, mental retardation and facial asymmetry. It is associated with calvarial thickening and hyperpneumatisation of the frontal sinuses. Ipsilateral cerebellar atrophy occurs if the insult occurs before the age of 1 month while contralateral cerebellar atrophy is seen when the insult occurs after the age of 1 month. ${ }^{1,2}$

\section{Case report}

A 6-year-old female child presented with giddiness for 4 days. She had a history of right-sided hemiparesis and right sided focal convulsions for $3 \frac{1}{2} 2$ years. Birth history was normal with full term normal vaginal delivery; baby cried immediately after birth with no neonatal intensive care unit (ICU) stay.

Magnetic resonance imaging (MRI) of the brain showed atrophy of the right cerebellar hemisphere with prominence of the cerebellar folia (Figure 1). Left cerebral peduncle was small in size suggestive of Wallerian degeneration (Figure 2). Left cerebral hemisphere was atrophic as evident from the prominence of sulcal spaces, sylvian fissure and ex-vacuo dilatation of the left lateral ventricle with the shift of midline structures towards left. An old infarct with gliosis was noted in left fronto-temporo-parietal region appearing hypointense on T1WI and hyperintense on T2WI and fluid attenuation inversion recovery with no restriction on diffusion weighted imaging. Associated uniform thickening of the calvarium was noted on the left side (Figures 3 and 4). Magnetic resonance angiography showed attenuation and reduced signal intensity of the M2 and M3 segments of the left middle cerebral artery (MCA) (Figure 5). A diagnosis of Davidoff-DykeMasson syndrome type III was made.

\section{Discussion}

Dyke-Davidoff-Masson syndrome is a rare condition derived from its researchers Dyke, Davidoff and Masson who first reported the condition in 1933. Changes of facial asymmetry, hemiparesis, seizures and mental retardation were seen in nine patients. Diffuse or subtotal cerebral hemiatrophy is the classical imaging finding. However, occasionally unilateral focal atrophy may be seen in the cerebral peduncle, thalami, parahippocampal region, pons and crossed cerebellar hemisphere. The clinical features of DDMS are mental retardation, focal or generalised seizures, learning disabilities and contralateral hemiparesis with an upper motor neuron type of facial palsy. Although there is no sex predilection and any side of the brain can be involved, male gender and involvement of left cerebral hemisphere are commonly found. ${ }^{2}$

There are two types, the infantile or congenital variety, and the acquired type. The infantile variety occurs because of gestational or neonatal vascular occlusion of the MCA, unilateral cerebral arterial circulation anomalies, coarctation of the mid-aortic arch or infections. Patients become 


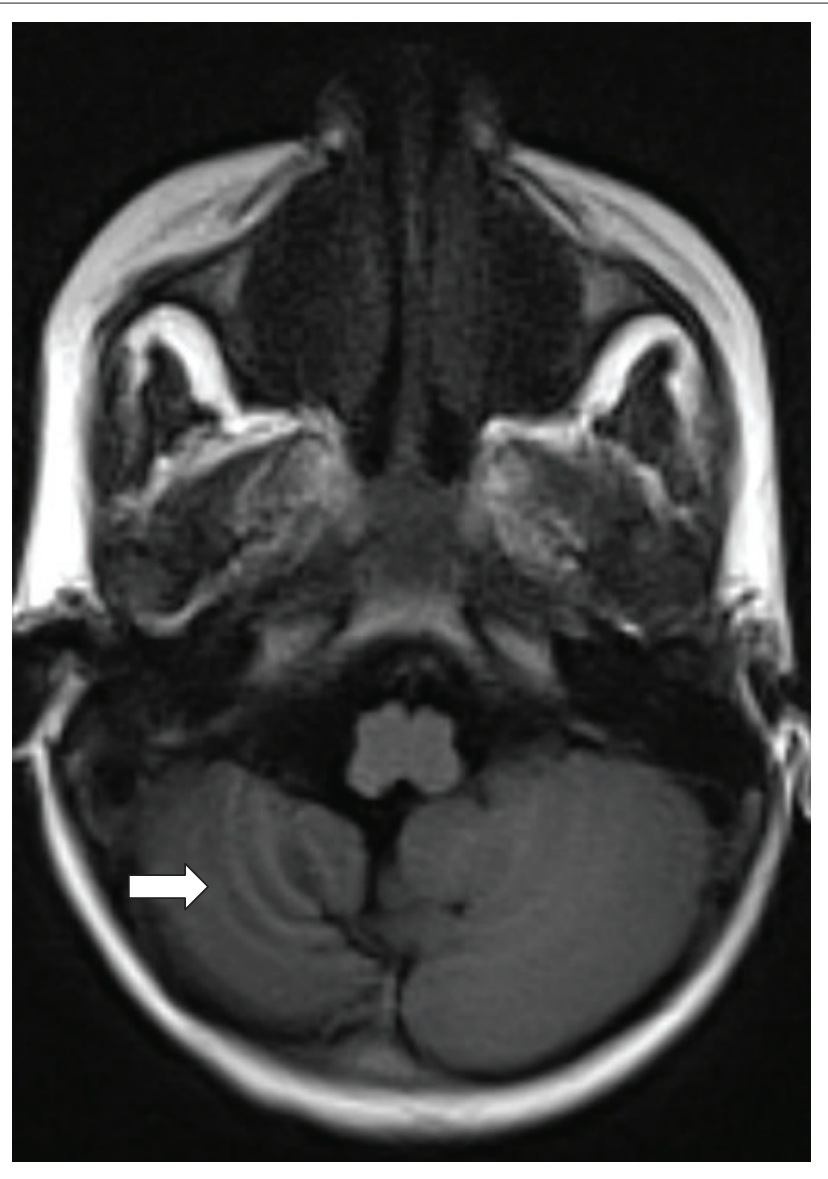

FIGURE 1: Axial T1 sequence showing right cerebellar atrophy with prominence of cerebellar folia (arrow).

symptomatic in the perinatal period or infancy. The acquired type results from prolonged febrile seizures, birth asphyxia, infection, ischemia, tumour and haemorrhage. The classical computed tomography (CT) and MRI findings of DDMS are seen only if the brain insult occurs before 3 years of age. The formation of brain sulci begin in the 4th month of gestation and usually complete by the end of the 8 th month. The maximum growth of a child's head occurs in the early years because of outward pressure of the enlarging brain on the cranial vault and reaches half of its adult size by the end of 1 year and $75 \%$ of the adult size by the end of 3 years. Hence only if brain damage occurs before 3 years of age, there is increased growth of the other structures overlying the brain, resulting in thickening of the cranial vault with increased width of diploic spaces, enlarged paranasal sinuses and an elevated orbital roof. Several ischemic episodes due to different causes lead to a reduction in the production of brain-derived neurotrophic factors with resultant cerebral atrophy. ${ }^{2}$

Dyke-Davidoff-Masson syndrome or cerebral hemiatrophy, is a congenital neonatal or early infantile condition. Mental retardation may not be always present. Seizures often occur months or years after the onset of hemiparesis. Speech or language disorder may be present. CT and MRI findings characteristically demonstrate parenchymal abnormality because of unilateral loss of cerebral volume with compensatory bone alterations in the adjacent calvarium. ${ }^{3}$

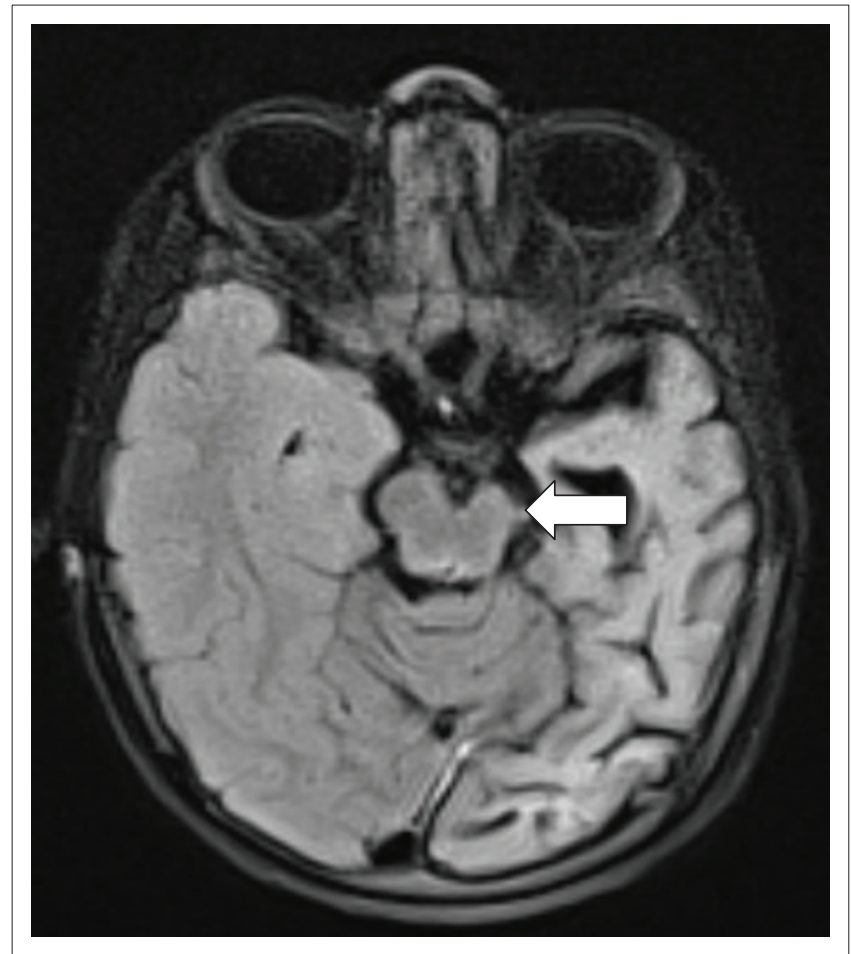

FIGURE 2: Axial fluid attenuation inversion recovery sequence showing small sized left cerebral peduncle because of Wallerian degeneration (arrow) with left cerebral hemiatrophy.

No prominent sulci are observed if the vascular insult occurs during embryogenesis (when the formation of gyri and sulci is incomplete). If the vascular insult occurs after sulcation is complete or after birth, then prominent sulci are seen. CT and MRI show gliosis, encephalomalacia, porencephaly, loss of gyri and white matter in the involved cerebral hemisphere with the prominence of the adjoining sulcal spaces and sylvian fissure. Ipsilateral enlargement of the lateral ventricle with midline shift towards the atrophic side is seen in the hemiatrophic brain. Ipsilateral internal capsule, thalamus and cerebral peduncle are small or hypoplastic secondary to Wallerian degeneration. Skull vault shows compensatory changes reflecting adaptations to the cerebral hemiatrophy. Thickening of the ipsilateral calvarium (diploic spaces and inner table), loss of convolutional markings in the inner table of the skull, elevation of the petrous ridge, orbital roof and sphenoid wing, overdevelopment of the frontal sinus, displacement of the falx attachment and diminished size of middle or anterior cranial fossa are usually seen. ${ }^{3}$

Three patterns of DDMS are seen on MRI. Pattern 1 shows diffuse cortical and subcortical atrophy. Pattern 2 shows diffuse cortical atrophy with porencephalic cysts, and pattern 3 shows previous infarction with gliosis in the middle cerebral artery (MCA) territory. The differential diagnosis for the triad of epilepsy, hemiplegia and hemiatrophy includes Sturge-Weber syndrome, Rasmussen's encephalitis and hemimegalencephaly. ${ }^{4}$ Ipsilateral or crossed cerebellar atrophy (CCA) is rarely reported in DDMS.

Ipsilateral cerebellar atrophy occurs if the insult occurs before the age of 1 month while contralateral cerebellar 

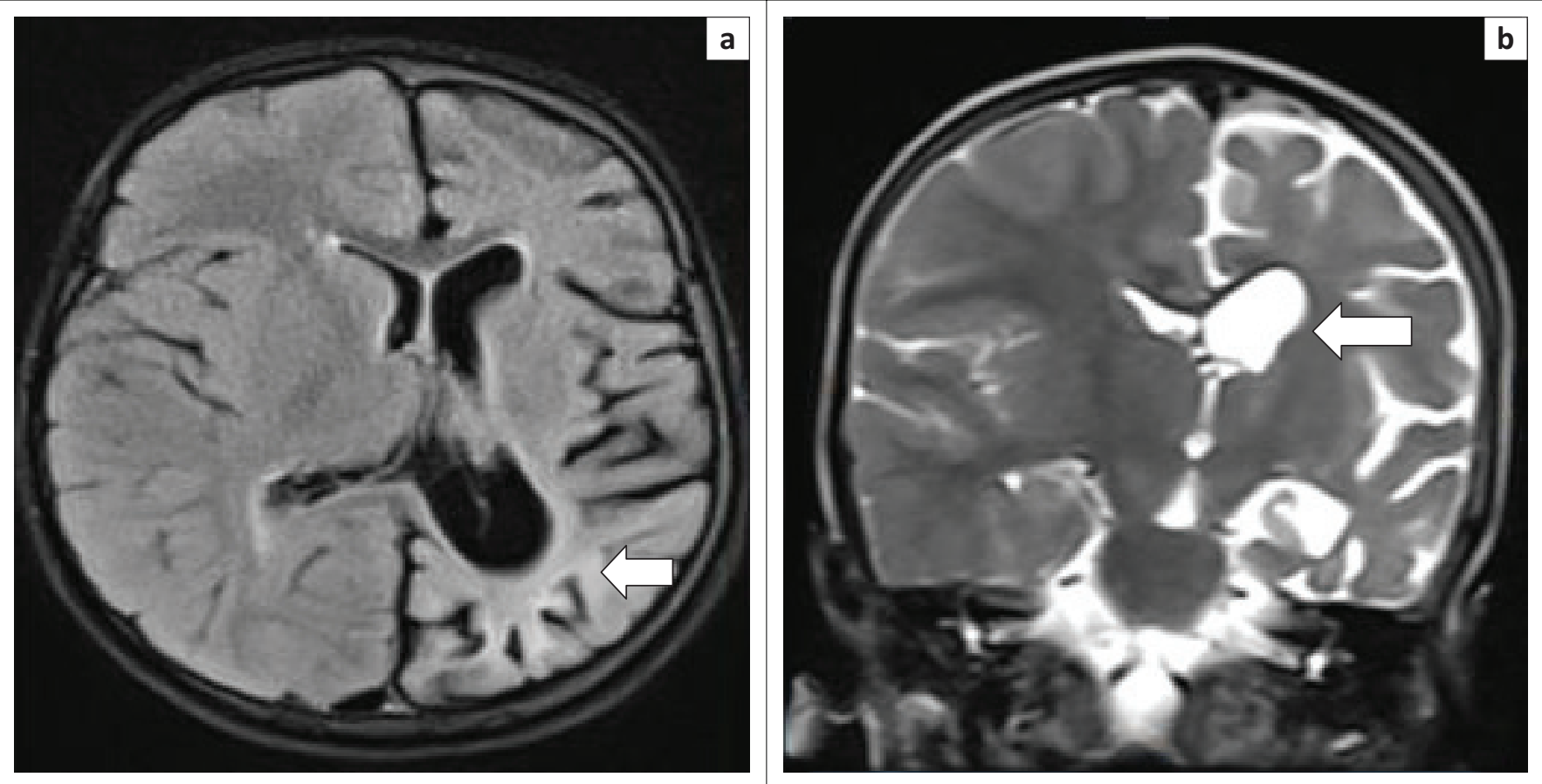

FIGURE 3: (a) Axial fluid attenuation inversion recovery sequence; (b) Coronal T2 sequence showing gliosis in left parieto-occipital region (arrow) with left cerebral hemiatrophy and ex-vacuo dilatation of the left lateral ventricle (arrow).

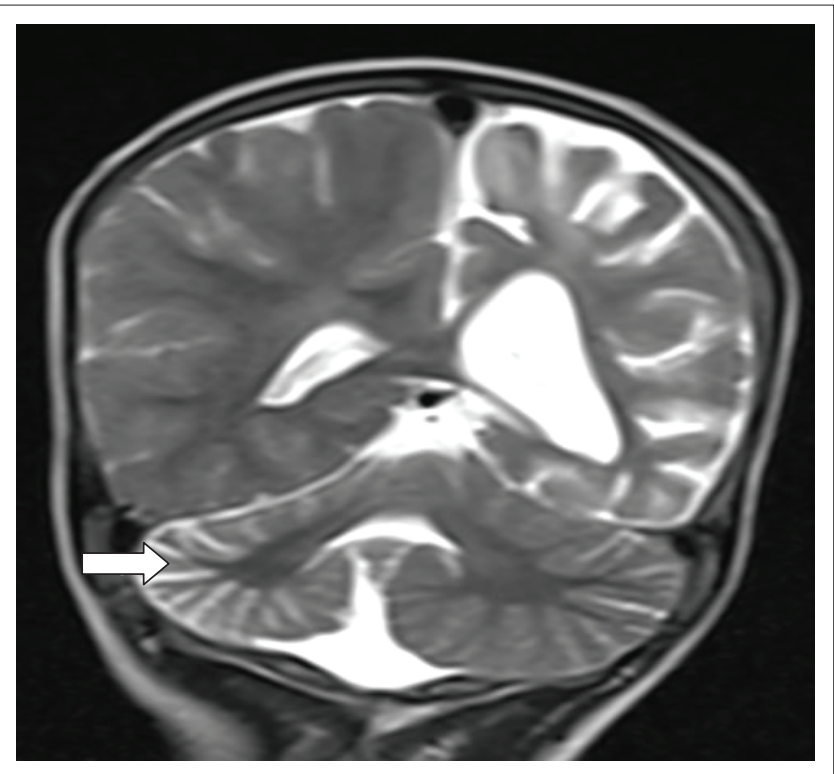

FIGURE 4: Coronal T2 sequence showing gliosis in the left parietal region with left cerebral hemiatrophy, ex-vacuo dilatation of the left lateral ventricle and right cerebellar atrophy (arrow).

atrophy is seen when the insult occurs after the age of 1 month. ${ }^{1}$ Crossed cerebellar diaschisis is due to contralateral cerebellar dysfunction because of a lesion in the ipsilateral cerebral hemisphere. It can be transient or permanent. Crossed cerebellar diaschisis or cerebellar atrophy occurs because of contralateral cerebellar hypometabolism secondary to a supratentorial insult. It is usually diagnosed by positron emission tomography (PET) imaging. However, it can also be diagnosed by perfusionweighted MRI before structural changes appear on conventional MRI. There is a functional disconnection of the cerebellar hemisphere from the cerebral cortex. Interruption

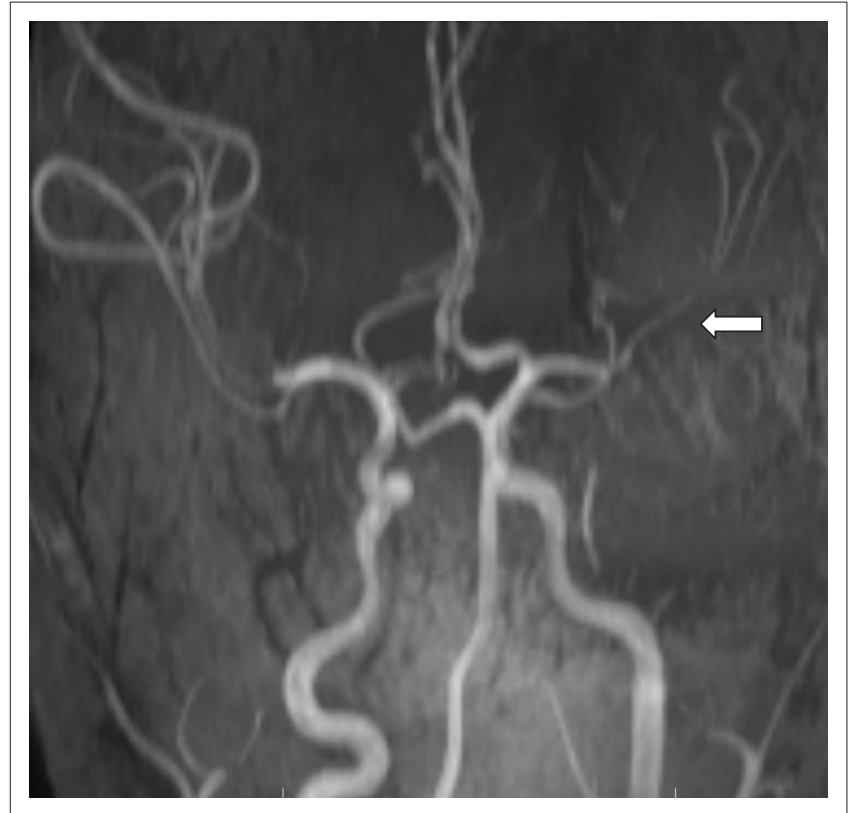

Source: Department of Radiology, Dr. D.Y. Patil Medical College, Hospital \& Research Centre

FIGURE 5: Magnetic resonance angiography of the brain showing attenuation and reduced signal intensity of M2 and M3 segments of left MCA (arrow).

of the cerebro-ponto-cerebellar pathway causes transneuronal metabolic depression. ${ }^{5}$ Diaschisis is because of transient impairment of functional activity in an area remote from the site of the primary brain lesion. There is a reduction in blood flow and oxygen uptake in the cerebellar hemisphere contralateral to the side of supratentorial ischemic insult. ${ }^{1}$ Cerebro-cerebellar diaschisis (CCD) can also be observed in the supratentorial infarcts, haemorrhage, arteriovenous malformation and tumour. CCD is initially a reversible functional process without structural change seen on PET 
scan and perfusion MRI. With the continuation of the insult, irreversible CCA occurs which can be detected on CT and MRI. CCA is rare and irreversible and is the continuum of the biological process seen in crossed cerebellar diaschisis. ${ }^{1}$ Reversible CCD and irreversible CCA belong to the same spectrum. CCD and CCA were mistakenly used interchangeably in the literature. ${ }^{6}$ Rarely diffuse cerebellar atrophy may occur in DDMS probably due to insufficiency in the posterior circulation. ${ }^{7}$

\section{Conclusion}

Dyke-Davidoff-Masson syndrome with ipsilateral or CCA is a rare entity, which can be diagnosed on CT and MRI. The timing of the insult can be predicted from the presence of ipsilateral or CCA.

\section{Acknowledgements Competing interests}

The authors declare that they have no financial or personal relationship(s) that may have inappropriately influenced them in writing this article.

\section{Authors' contributions}

S.C and S.M.K. were responsible for the diagnosis of case, review of literature and preparation of case report. S.M.K. was responsible for the preparation of case report as per journal's requirement. A.M.P. and S.G.G. provided guidance for making the case report.

\section{References}

1. Gupta R, Joshi S, Mittal A, Luthra I, Mittal P, Verma V. Magnetic resonance imaging depiction of acquired Dyke-Davidoff-Masson syndrome with crossed cerebrocerebellar diaschisis: Report of two cases. J Pediatr Neurosci. 2015;10(3):294-296. https://doi.org/10.4103/1817-1745.165730

2. Roy U, Panwar A, Mukherjee A, Biswas D. Adult presentation of Dyke-DavidoffMasson syndrome: A case report. Case Rep Neurol. 2016;8(1):20-26.

3. Aguiar PH, Liu CW, Leitão $\mathrm{H}$, et al. MR and $\mathrm{CT}$ imaging in the Dyke-DavidoffMasson syndrome. Report of three cases and contribution to pathogenesis and Masson syndrome. Report of three cases and contribution to
differential diagnosis. Arq Neuropsiquiatr. 1998;56(4):803-807.

4. Sharma S, Singh S, Bhagat S, Garcha H. Dyke-Davidoff-Masson syndrome/ cerebral hemiatrophy in an adult male: A rare imaging case. Indian J Appl Radiol. 2016;2(1):1-3.

5. Narra R, Kamaraju SK, Pasupaleti B, Jukuri NR. Dyke-Davidoff-Masson syndrome: A case report of cerebral hemiatrophy with crossed cerebellar diaschisis. Afr Med Health Sci. 2015;14(2):150-152. https://doi.org/10.4103/2384-5589.170195

6. Algahtani HA, Aldarmahi AA, Al-Rabia MW, Young GB. Crossed cerebro-cerebellar atrophy with Dyke-Davidoff-Masson syndrome. Neurosciences (Riyadh). 2014;19(1):52-55.

7. Gökçe E, Beyhan M, Sade R. Radiological imaging findings of Dyke-DavidoffMasson syndrome. Acta Neurol Belg. 2017:1-9. 